\title{
Force-Extension Relations for Polymers with Sliding Links
}

\author{
Ralf Metzler, ${ }^{1,2, \text { f Yacov Kantor, }}$, 1, 用 and Mehran Kardar ${ }^{1}$ \\ ${ }^{1}$ Department of Physics, Massachusetts Institute of Technology, Cambridge, Massachusetts 02139 \\ ${ }^{2}$ NORDITA, Blegdamsvej 17, DK-2100 Copenhagen Ø, Denmark \\ ${ }^{3}$ School for Physics and Astronomy, Tel Aviv University, Tel Aviv 69978, Israel
}

(Dated: November 4, 2018)

\begin{abstract}
Topological entanglements in polymers are mimicked by sliding rings (slip-links) which enforce pair contacts between monomers. We study the force-extension curve for linear polymers in which slip-links create additional loops of variable size. For a single loop in a phantom chain, we obtain exact expressions for the average end-to-end separation: The linear response to a small force is related to the properties of the unstressed chain, while for a large force the polymer backbone can be treated as a sequence of Pincus-de Gennes blobs, the constraint effecting only a single blob. Generalizing this picture, scaling arguments are used to include self-avoiding effects.
\end{abstract}

PACS numbers: 05.40.Fb 64.60.Fr 82.35.Lr 87.15.La

Entanglements play an important role in the behavior of macromolecules. For instance, mechanical links (e.g., in catenanes) and knots naturally appear in long polymers [1]. In biological systems, specific proteins act upon topological states: the degree of entanglement of chromosomes during cell division [2], or knotted states in bacterial DNA which may arise during random ring closure [3], can be modified by topological enzymes [ 1 . Synthetic RNA trefoil knots have been used to prove the existence of a similar, previously unknown, topologychanging enzyme [5]. Tight molecular knots have even been found deep inside the native state of proteins [6]. Experimental advances now make it possible to manipulate single molecules by optical tweezers. Thus, tight knots could be tied into single actin filaments or DNA strands [7]. Mechanical properties, and forces in the $\mathrm{pN}$ range relevant to biopolymeric processes, can be measured by atomic force microscopy, or more direct micromechanical methods [8]. It is therefore possible to record the force-extension (FE) curve of single polymers with a fixed topology, from which valuable information about the properties of a molecule can be obtained and compared to theoretical predictions.

While there has been extensive progress in the statistical mechanics of polymers in the last decades [9, 10, the analysis of topological constraints is hampered by the difficulty of treating the resulting division of phase space into accessible and inaccessible regions. Since the mathematical methods of knot detection using topological invariants [11] cannot be conveniently incorporated into a statistical-mechanical formulation, one may try to use geometrical constrictions to mimic knots: Consider a polymer threaded through a small ring as depicted in Fig. 1, and not allowed to withdraw from it, although the ring may freely slide along the polymer and the loop size can change. Constrictions of this type (called slip-links

\footnotetext{
*Electronic address: metz@nordita.dk

${ }^{\dagger}$ Electronic address: kantor@post.tau.ac.i
}

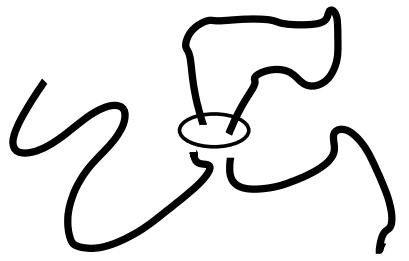

FIG. 1: Polymer threaded through a slip-link (ring) forms a loop and two dangling ends. It is not allowed to withdraw from the slip-link.

(SLs)) were introduced by Ball, Doi, Edwards and others 12] to investigate the elasticity of rubber, where they were used to represent entanglements between different polymers. Recently, a detailed study of the size distribution of loops in SL structures was performed [13]. In this work we consider FE curves of such polymers, with or without self-avoidance. We show that the knowledge of the statistics in the absence of the force, combined with the Pincus-de Gennes 114 blob picture, suffices to understand many features of the FE curves.

We first discuss the statistical effects of the slip-link for non-self-avoiding chains (phantom polymers). Consider an $N$-step chain with mean-squared step size $a^{2}$ in $d$ dimensional space. In the $N \gg 1$ limit, the probability density function for the end-to-end distance (EED) $\mathbf{r}$ is

$$
p_{N}(\mathbf{r})=\left(\frac{d}{2 \pi N a^{2}}\right)^{d / 2} \exp \left(-\frac{d r^{2}}{2 N a^{2}}\right)
$$

This expression also describes an $N$-step random walk on a (hyper)cubic lattice with lattice constant $a$. The number of closed $N$-step loops on such a lattice is $(2 d)^{N}[d /(2 \pi N)]^{d / 2}$. Now consider the case where a SL forces a closed loop of size $n$. Since the loop can be located on any of $N-n$ positions on the $N$ step chain, the number of possible configurations of the combined system of the polymer and the SL is $(N-n)(2 d)^{N-n}(2 d)^{n}[d /(2 \pi n)]^{d / 2}$. Thus, for $n \gg 1$ and 
$(N-n) \gg 1$, the probability for a given $n$ is

$$
g_{N}(n)=\mathcal{N}(N-n) n^{-d / 2}
$$

where $\mathcal{N}$ is a normalization factor. For $d>2, \mathcal{N}$ depends on the short-distance cut-off, i.e., on the microscopic details of the walk 15. The presence of the SL also modifies the probability density of the $\mathrm{EED}, p(\mathbf{r})$, by reducing the length of the backbone to $(N-n)$.

When the end-points of a polymer are stretched by a force $\mathbf{f}$, its properties can be derived from the partition function

$$
\mathcal{Z}(f)=\int d^{d} \mathbf{r} p(\mathbf{r}) e^{\mathbf{f} \cdot \mathbf{r} / T},
$$

where $f=|\mathbf{f}|$, and the temperature $T$ is in energy units, i.e., $k_{B}=1$. In the presence of the force, the vector $\mathbf{r}$ is on average parallel to $\mathbf{f}$, and from Eq. (3), its mean projection along $\mathbf{f}$ is given by

$$
\langle r\rangle_{f}=T \frac{\partial \ln \mathcal{Z}}{\partial f} .
$$

In particular, for $p(\mathbf{r})=p_{N}(\mathbf{r})$ in Eq. (11), the partition function is $\mathcal{Z}=\exp \left[N a^{2} f^{2} /\left(2 d T^{2}\right)\right]$, while the mean EED is a linear function of $f,\langle r\rangle_{f}=f N a^{2} /(d T)$, for any arbitrary value of the force. Since the mean-squared EED $R^{2}$ of an unstrained (zero-force) phantom chain is $N a^{2}$, the FE relation can be re-written as

$$
\langle r\rangle_{f}=\frac{R^{2}}{d T} f .
$$

More complicated forms of $p(\mathbf{r})$ do not lead to a simple linear relation, and in many cases the relation between $\langle r\rangle_{f}$ and an arbitrary $f$ cannot be calculated exactly. However, for sufficiently small $f$, linear response theory provides a simple universal answer: By expanding the exponent in Eq. (3) in powers of $f$, and by omitting powers higher than 2, we see that Eq. (5) is valid for arbitrary spherically symmetric $p(\mathbf{r})$, provided that $R^{2}$ is the mean squared EED calculated at zero force. The force can be considered small when $\langle r\rangle_{f} \ll R$, i.e., for $f \ll T / R$.

The probability density of the EED of a phantom chain with a simple SL (Fig. 11) is given by

$$
p(\mathbf{r})=p_{N-n}(\mathbf{r}) g_{N}(n)
$$

(see Eqs. (1) and (2)). Here, $N-n$ is the number of monomers in the force-carrying backbone of the polymer. Thus, $R^{2}$ can be found by integrating $r^{2}$ with the above statistical weight, over all possible $\mathbf{r}$ and $n$, leading to

$$
R^{2}=a^{2}\left(N-\langle n\rangle_{0}\right),
$$

where

$$
\langle n\rangle_{0}=\left\{\begin{array}{ll}
c_{d} & \text { for } d>4 \\
c_{4} \ln N & \text { for } d=4 \\
c_{d} N^{2-d / 2} & \text { for } 2<d<4 \\
\frac{1}{2} \frac{N}{\ln N} & \text { for } d=2 \\
\frac{2-d}{6-d} N & \text { for } d<2
\end{array} .\right.
$$

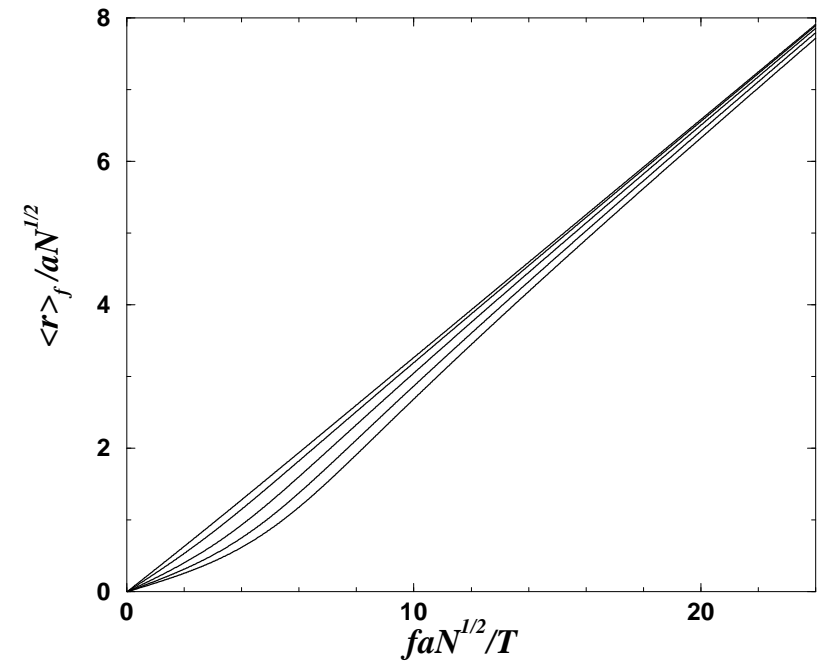

FIG. 2: Force-extension curves for a 100-monomer polymer with a slip-link in $d=3$ with its loop threaded through additional (from top to bottom) $m=0,1,2,3$ and 4 , rings.

Here, $c_{d}$ are short length-scale cutoff-dependent constants. In this expression, $\langle n\rangle_{0}$ is simply the mean number of monomers inside the constricted loop, and $R^{2}$ is obtained by replacing $N$ in the expression for $R^{2}$ of a simple phantom chain by $N-\langle n\rangle_{0}$. We note that for $d>2,\langle n\rangle_{0}$ has a sub-linear dependence on $N$, and as $d$ increases the correction created by the SL depends more weakly on $N[16]$. For $d>4$ random walks do not form long loops and $\langle n\rangle_{0}$ becomes independent of $N$.

The expression for $R^{2}$ of a phantom chain with a SL can now be substituted in Eq. (5), to obtain the FE relation $\langle r\rangle_{f}=f\left(a^{2} / d T\right)\left(N-\langle n\rangle_{0}\right)$ for small $f$. For large $f$, this expression is no longer valid. However, by direct inspection of the required average we find that Eqs. (3), (4), and (6) lead to

$$
\begin{aligned}
\langle r\rangle_{f} & =T \frac{\partial}{\partial f} \ln \int d n g_{N}(n) \exp \left[\frac{f^{2} a^{2}(N-n)}{2 d T^{2}}\right] \\
& =\frac{a^{2}}{d T}\left(N-\langle n\rangle_{f}\right) f,
\end{aligned}
$$

where $\langle n\rangle_{f}$ is the mean loop size in the presence of the force, equal to

$$
\langle n\rangle_{f}=\frac{\int_{n_{0}}^{N} d n n g_{N}(n) \exp \left[-f^{2} a^{2} n /\left(2 d T^{2}\right)\right]}{\int_{n_{0}}^{N} d n g_{N}(n) \exp \left[-f^{2} a^{2} n /\left(2 d T^{2}\right)\right]}
$$

The lower limit of $n_{0}$ in the above integrals is the minimal loop size allowed by the specific model.

The FE relation is no longer linear, even for a phantom polymer with one SL, although the deviation disappears rapidly with increasing $f$. For the simple SL with the weight in Eq. (2), the nonlinearity is barely detectable even for the relatively small value of $N=100$, as indicated by the top line in Fig. 2. In more complicated topologies of many SLs, we frequently encounter 


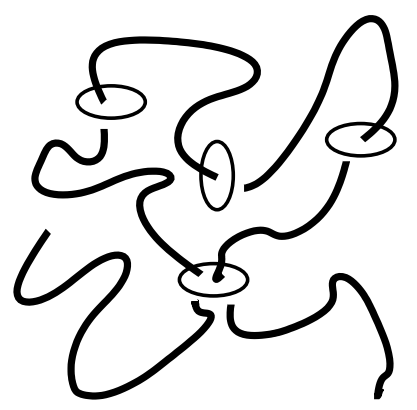

FIG. 3: A slip-link whose loop is threaded through an additional $m=3$ rings which are restricted to stay on the loop.

the behavior $g_{N}(n) \sim n^{-\alpha}$ [13], where $n$ is the total number of monomers that do not belong to the direct path between the ends of the polymer, while $N$ appears in the prefactor or other non-singular parts of the probability density. In such cases, the non-linearities become more pronounced as $\alpha$ decreases. Let us specifically consider the "toy-example" of a SL in which $m$ additional rings slide around the loop, as depicted in Fig. 3. The number of ways of placing these sliding rings leads to $g_{N}(N)=\mathcal{N}(N-n) n^{m-d / 2}$ [17]. Depending on the values of $m$ and $d$, three different behaviors can be distinguished:

(i) For $m>d / 2-1$, the integrals in Eq. (9) are dominated by large $n$. We can thus set the lower limit of the integrals to 0 , and introduce the new variable $x=n / N$, to get

$$
\frac{\langle r\rangle_{f}}{a \sqrt{N}}=\frac{f a \sqrt{N}}{T \sqrt{2 d}}\left[1-\frac{\int_{0}^{1} d x x^{m+1-d / 2} \mathrm{e}^{-x f^{2} a^{2} N /\left(2 d T^{2}\right)}}{\int_{0}^{1} d x x^{m-d / 2} \mathrm{e}^{-x f^{2} a^{2} N /\left(2 d T^{2}\right)}}\right] .
$$

This FE curve now satisfies a scaling form $\langle r\rangle_{f} / R=$ $\Phi(f R /[\sqrt{2 d} T])$, where the scaling function has the limits

$$
\Phi(z) \sim \begin{cases}z /(1+m-d / 2), & z \rightarrow 0 \\ z-c / z, & z \rightarrow \infty .\end{cases}
$$

The initial slope is reduced for larger $m$, as depicted in Fig. 2, while the asymptotic form at large $f$ is reached with a correction that falls off as $1 / f$. The physical origin of the nonlinearity is the tightening of the initially large loop in the intermediate regime.

(ii) For $d / 2-2>m>d / 2-1$, the mean loop size grows as $N^{2+m-d / 2}$, playing the role of an additional (sub-leading) length scale. Consequently, the FE curve no longer has a simple scaling form, and behaves as

$$
\frac{\langle r\rangle_{f}}{R}=\frac{f R}{\sqrt{2 d} T}\left[1-N^{2+m-d / 2} \phi\left(\frac{f R}{T}\right)\right] .
$$

This case may be most similar to that of knots in three dimensions, and indeed such corrections to scaling were used in Ref. [18] to extract the size of the knot.

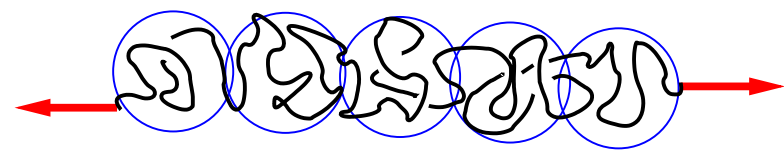

FIG. 4: In the Pincus-de Gennes scenario, the stretched polymer is viewed as a linear sequence of "blobs" (circumscribed by circles). Within each blob the polymer is unstressed; the size and number of blobs depends on the stretching force.

(iii) For $m<d / 2-2$, both integrals in Eq. (9) are dominated by the short distance cutoff, resulting in $\langle n\rangle_{f} \approx n_{0}$, independent of $f$. The $\mathrm{FE}$ curve is thus linear in this regime, with finite size corrections that disappear as $1 / N$.

While the above results are easily obtained for a phantom polymer with a SL, it is convenient to restate them in a form that is more generally valid, and, in particular, applicable to interacting polymers. This will be done using the Pincus-de Gennes picture [14, according to which a stretched polymer (without a SL) at short scales does not feel the influence of the external force, and correlations remain as in the unforced polymer, while at longer distances it is essentially a linear object aligned to the force. The polymer can then be visualized as a linear chain of blobs, as depicted in Fig. 4 . The number of monomers $N_{b}$ inside a blob is determined by the condition $f R_{b} \approx T$, where $R_{b}$ is the EED of $N_{b}$ monomers. In the case of a phantom polymer $\left(R_{b}=a N_{b}^{1 / 2}\right)$, this leads to $N_{b} \approx(T / f a)^{2}$, while in the more general case with $R_{b}=a N_{b}^{\nu}$, we get $N_{b} \approx(T / f a)^{1 / \nu}$. Consequently, for large forces the EED of a whole polymer is the size of a single blob times the number of blobs, i.e.,

$$
\langle r\rangle_{f}=\left(N / N_{b}\right) R_{b}=a N N_{b}^{\nu-1}=a N(f a / T)^{\frac{1}{\nu}-1} .
$$

For phantom polymers the FE curve remains linear even for large $f$, while for a self-avoiding polymer in $d=3$ (with $\nu \approx 0.58$ ) the relation is highly non-linear.

We now note that $N_{b}$ is the scale over which the exponential factor in Eq. (9) decays, and consequently the mean size of the SL loop for $f \gg T /(a \sqrt{N})$ can be estimated as

$$
\langle n\rangle_{f} \approx \frac{\int_{n_{0}}^{N_{b}} d n n g_{N}(n)}{\int_{n_{0}}^{N_{b}} d n g_{N}(n)} .
$$

However, this expression is exactly the size of the link in a polymer consisting of $N_{b}$ monomers in the absence of an external force, i.e.

$$
\langle n\rangle_{f, N} \approx\langle n\rangle_{0, N_{b}} .
$$

The first subscript in this equation denotes the size of the force, while the second index indicates the total number of monomers. We can, therefore, view the SL loop as being confined to a single blob. Since within a blob the external force is not felt, its size is determined by regarding the entire polymer length as $N_{b}$, as depicted qualitatively in Fig. 5 . 


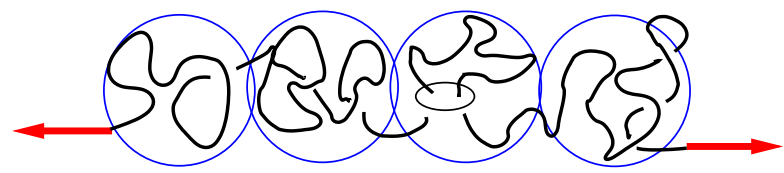

FIG. 5: Qualitative representation of a stretched polymer with a SL. The loop created by the SL is contained within a single blob, and its size is determined only by the number of the monomers in the blob.

While the leading term in the expression for the EED of a strongly stretched polymer with a SL will still have a form given by Eq. (13), subleading corrections depend on the influence that the presence of SL has on the EED of the unstretched polymer. For a phantom chain there is a clear separation between the segment that creates the link and the remainder of the chain, and, consequently, in the absence of an external force, the reduction in $R^{2}$ can be simply related to the reduction of $N$ by Eq. (7). This does not have to be the case in the presence of interactions, and each case must be considered separately. In the presence of self-avoiding interactions (for $d<4$ ), it can be shown [13], that for $n \ll N$ the statistical weight of an $n$-monomer loop is given by $g_{N}(n) \sim n^{-c}$, where $c>2$. Consequently, $\langle n\rangle_{0}$ is independent of $N$, and will cause no detectable modification in the $\mathrm{FE}$ curve of a self-avoiding polymer. This picture can be easily generalized to a sequence of (non-interpenetrating) SLs. At zero force, the loops will compete for the available length, each acquiring a fraction of the overall length, as described in Ref. 13]. At strong force, in the blob regime, each slip link is most likely confined to its own blob.

Viewing the stretched polymer as a sequence of blobs, with only individual blobs affected by the presence of the constraints such as SLs, creates a convenient framework for evaluation of FE relations. This picture may possibly be extended to knotted polymers: If the size of each prime knot factor depends on the number of monomers $N$ as a power law $N^{t}$, then the application of a strong stretching force will confine the knot to a blob and will reduce its size to $N_{b}^{t}$. Such a scenario (at small forces) was explored in a recent Monte Carlo study [18].

Acknowledgments: We thank A. Hanke and O. Farago for helpful discussions. This work was supported by USIsrael Binational Science Foundation (grant 1999-007), and by the National Science Foundation (grants DMR01-18213 and PHY99-07949). RM acknowledges financial support from the Deutsche Forschungsgemeinschaft.
[1] H. L. Frisch, and E. Wasserman, J. Am. Chem. Soc. 83, (1961); K. Mislow, Introduction to Stereochemistry, Benjamin, New York (1965); G. Schill, Catenanes, Rotaxanes, and Knots, Academic, New York (1971); D. M. Walba, Tetrahedron 41, 3161 (1985); Catenanes, Rotaxanes and Knots, edited by J.-P. Sauvage and C. DietrichBuchecker, VCH, Weilheim (1999).

[2] B. Alberts, K. Roberts, D. Bray, J. Lewis, M. Raff and J. D. Watson, The molecular biology of the cell (Garland, New York, 1994).

[3] V. V. Rybenkov N. R. Cozazarelli, A. V. Vologodskii, Proc. Natl. Acad.Sci. USA 90, 5307 (1993); S. Y. Shaw and J. C. Wang, Science 260, 533 (1993).

[4] W. R. Bauer, F. H. C. Crick, and J. H. White, Sci. Am. 243, 118 (1980); N. R. Cozzarelli, S. J. Spengler, and A. Stasiak, Cell 42, 325 (1985); S. A. Wasserman and N. R. Cozzarelli, Science 232, 951 (1986).

[5] H. Wang R. J. Di Gate and N. C. Seeman, Proc. Nat. Acad. Sci. (USA) 93, 9477 (1996).

[6] W. R. Taylor, Nature 406, 916 (2000); R. Takusagawa and K. Kamitori, J. Am. Chem. Soc. 118, 8945 (1996).

[7] T. Arai, R. Yasuda, K.-i. Akashi, Y. Harada, H. Miyata, K. Kinosita, Jr., and H. Itoh, Nature 399, 446 (1999).

[8] M. Rief, M. Gautel, F. Oesterhelt, J. M. Fernandez and H. E. Gaub, Science 276, 1109 (1997); S. B. Smith, L. Finzi, and C. Bustamante, Science 258, 1122 (1992); B. Smith, Y. Cui, and C. Bustamante, Science 271, 795 (1996); K. Svoboda, S. M. Block, Ann. Rev. Biophys. Biomol. Structure 23, 247 (1994); A. Ashkin, Proc. Natl. Acad. Sci. USA 94, 4853 (1997); H. G. Hansma, J. Vac. Sci. Technology B14, 1390(1995); K. Berg-Sorensen and H. Flyvbjerg (unpublished).

[9] P. G. de Gennes, Scaling Concepts in Polymers Physics
(Cornell University Press, Ithaca, New York, 1979)

[10] M. Doi and S. F. Edwards, The Theory of Polymer dynamics (Clarendon Press, Oxford, 1986).

[11] J. W. Alexander, Trans. Amer. Math.Soc. 30, 275 (1928). V. F. R. Jones, Bull. Am. Math. Soc. 12, 103 (1985); and Pacific J. Math. 137, 311 (1989). P. Freyd, D. Yetter, J. Hoste, W. B. R. Lickorish, K. C. Millett and A. Ocneanu, Bull. am. Math. Soc. 12, 103 (1985). L. H. Kauffman, Topology 26, 395 (1987).

[12] M. Doi and S. F. Edwards, J. Chem. Soc. Faraday Trans. 2 74, 1802 (1978); R. C. Ball, M. Doi, S. F. Edwards, and M. Warner, Polymer 22, 1010 (1981); D. Adolf, Macromolecules 21, 228 (1988); M. Kosc, Colloid Polym. Sci. 266, 105 (1988); P. G. Higgs and R. C. Ball, Europhys. Lett. 8, 357 (1989).

[13] R. Metzler, A. Hanke, P. G. Dommersnes, Y. Kantor, M. Kardar, Phys. Rev. Lett. 88, 188101 (2002); Phys. Rev. E (2002), in press.

[14] P. Pincus, Macromol. 9, 386 (1976); P. G. de Gennes, P. Pincus, R. M. Velasco, F. Brochard, J. Phys. (Paris), 37 1461 (1976).

[15] For $d=2, \mathcal{N}=1 / N \ln N$, while for $d<2, \mathcal{N}=[(2-$ d) $(4-d) / 4] N^{(d / 2)-2}$.

[16] The numerical prefactor $c_{d}$ will be of order unity when the diameter $b$ of the SL is of the same order of magnitude as the elementary step size $a$. For $b>a$, the prefactor will be of the order $(b / a)^{d-2}$, for $2<d<4$.

[17] The short-distance scaling behavior of this example is in fact the same as with $m$ additional slip links sliding on the central loop 13.

[18] O. Farago, Y. Kantor, and M. Kardar, preprint (2002). 